\title{
Motivação de alunos do ensino fundamental: relações entre rendimento acadêmico, autoconceito, atribuições de causalidade e metas de realização*
}

Melissa Picchi Zambon

Tânia Maria Santana de Rose

Universidade Federal de São Carlos

Contato:

Tânia Maria Santana de Rose

Universidade Federal de São Carlos

Departamento de Psicologia

Centro de Educação e Ciências Humanas

São Carlos/SP

tmsrose@terra.com.br

\section{Resumo}

Este estudo insere-se no âmbito da pesquisa que aborda a influência exercida por construtos motivacionais de natureza cognitiva - tais como crenças, metas e valores dos estudantes - sobre a motivação e o rendimento acadêmico. Procurou-se identificar as relações entre níveis baixo, médio e alto de rendimento na disciplina de português e o autoconceito, as atribuições de causalidade para sucesso e fracasso e as metas de realização apresentadas por estudantes do ensino fundamental. Um grupo de 159 alunos de $6^{\text {a }}$ e $7^{\text {a }}$ séries responderam a três instrumentos específicos para avaliação dos construtos motivacionais. Foram encontradas diferenças significativas quanto ao autoconceito, às atribuições e às metas apresentadas pelos os alunos com alto rendimento e os resultados demonstrados por alunos com baixo e médio rendimento, sendo que os alunos com alto rendimento apresentaram autoconceito em leitura e escrita mais elevado, atribuíram mais o sucesso à capacidade e ao esforço e tiveram maior tendência à adoção da meta aprender do que os alunos com notas médias e baixas. Discutem-se os resultados como indicadores da qualidade da motivação dos estudantes e do engajamento motivacional, bem como a importância da influência do contexto de sala de aula para a compreensão e a promoção da motivação dos estudantes.

\section{Palavras-chave}

Motivação no ensino fundamental - Construtos motivacionais Rendimento acadêmico.

* Apoio financeiro: CAPES. 


\title{
Student motivation in fundamental education: relations between academic performance, self-image, attributions of causality and goals*
}

Melissa Picchi Zambon

Tânia Maria Santana de Rose

Universidade Federal de São Carlos

\begin{abstract}
This study is part of a research that focuses on the influence exerted by motivational constructs of a cognitive nature - such as students' beliefs, goals and values - upon their motivation and academic performance. We have tried to identify the relations between low, average, and high levels of performance in the discipline of Portuguese language and the factors of self-image, attributions of causality towards success or failure, and goals described by students of fundamental education. A group of 159 pupils from the $6^{\text {th }}$ and $7^{\text {th }}$ grades answered to three specific instruments designed to evaluate motivational constructs. Significant differences were found related to self-image, to the attributions, and to the goals described by the pupils with high-performance against those demonstrated by pupils with average and low performance, with high-performance pupils presenting better self-image in reading and writing, attributing success more to ability and effort, and having stronger tendency to adopt the goal of learning, when compared to pupils with average and low grades. The results are discussed as indicators of the quality of the pupils' motivation and of their motivational commitment, as well as the importance of the influence of the classroom context upon pupils' understanding and the promotion of their motivation.
\end{abstract}

\section{Keywords}

Motivation in fundamental education - Motivational constructs Academic performance.

\section{Contact:}

Tânia Maria Santana de Rose

Universidade Federal de São Carlos

Departamento de Psicologia

Centro de Educação e Ciências Humanas

São Carlos/SP

tmsrose@terra.com.br

* This work has been supported by CAPES. 
A pesquisa sobre motivação no contexto escolar fundamentada nas teorias cognitivo-sociais tem produzido um sólido conjunto de evidências que demonstram como as crenças e metas dos alunos afetam a aquisição de novas aprendizagens, o engajamento cognitivo, o aproveitamento acadêmico e a apresentação de comportamentos, estratégias e habilidades já aprendidas (PINTRICH, 2003).

A motivação para a realização acadêmica é entendida como um processo complexo que estimula, direciona e sustenta o comportamento voltado para o alcance de uma meta, resultante da convergência de aspectos cognitivos e contextuais. A natureza multifacetada, mutável e sensível à influência do contexto de sala de aula e da escola vem sendo amplamente aceita conceitualmente e demonstrada empiricamente (BZUNECK, 2009a; ECCLES; WIGFIELD, 2002; PINTRICH, 2003; PINTRICH; SCHUNK, 2002).

Dentre as inúmeras contribuições derivadas dos estudos realizados pelas diferentes teorias cognitivo-sociais, pode-se destacar a demonstração de como diferentes aspectos cognitivos - ou seja, as crenças e metas dos alunos relativas à aprendizagem - influenciam a motivação e o rendimento acadêmico. A fim de se entender a motivação para a realização acadêmica, devem ser levadas em conta as múltiplas facetas relacionadas às crenças dos estudantes sobre o que pensam a respeito de si mesmos como aprendizes, suas crenças a respeito de seu valor, suas capacidades (autoeficácia e autoconceito), suas explicações quanto ao sucesso e ao fracasso (atribuições de causalidade), suas metas (metas de realização) e seus sentimentos em relação à escola, à aprendizagem, às matérias e aos professores (BZUNECK, 2009a; PINTRICH, 2003; WIGFIELD et al., 2006).

Um segundo conjunto de contribuições refere-se às evidências sobre o papel dos aspectos do contexto de sala de aula nas crenças e metas motivacionais, bem como na aprendizagem e no rendimento acadêmico. Conta-se com um conhecimento consistente sobre como os professores e as práticas de ensino podem influenciar a motivação dos alunos tanto de forma positiva como negativa (AMES, 1992; GRAHAM; WEINER, 1996; PINTRICH, 2003; STIPEK, 1996).

As relações entre o rendimento acadêmico e o autoconceito acadêmico, as atribuições de causalidade e as metas de realização têm merecido a atenção dos pesquisadores, dado o potencial para auxiliar a compreensão do papel da motivação na promoção de resultados educacionais positivos e negativos.

\section{Autoconceito acadêmico}

0 autoconceito refere-se ao conjunto de autopercepções da pessoa formado a partir de suas experiências e das interpretações do ambiente, sendo fortemente influenciado por apreciações e reforços fornecidos pelos outros significativos (SHAVELSON; BOLUS, 1982). 0 autoconceito é concebido como tendo uma estrutura multidimensional e específica para diferentes áreas da vida, como a dimensão acadêmica, o âmbito social, a aparência e a capacidade física (MARSH, 1990b; MARSH; KÖLLER, 2003; PEIXOTO; ALMEIDA, 2011).

A relação entre autoconceito acadêmico e rendimento tem sido investigada com base em uma abordagem empírica que evidencia o princípio de que as pessoas buscam ativamente manter a consistência entre como se percebem e como se comportam, sendo que pessoas com percepções positivas de si próprias esforçam-se para realizarem as tarefas de maneira que sejam mais consistentes com suas autopercepções (MARSH, 1984,1986, 1990b; MARSH; KÖLLER, 2003).

Tem sido consistentemente verificado que alunos de ensino fundamental, médio e superior que atingem um alto rendimento acadêmico têm maior probabilidade de apresentarem elevado autoconceito acadêmico e vice-versa, o que evidencia uma relação de reciprocidade entre autoconceito e realização acadêmica, ou seja, se, por um lado, o sucesso ou o fracasso escolar em um domínio acadêmico contribui 
para a determinação do nível de autoconceito, por outro, o nível de autoconceito influencia os processos relacionados à aprendizagem e, consequentemente, o rendimento acadêmico (CARNEIRO; MARTINELLI; SISTO, 2003; CIA; BARHAM, 2008; MARSH, 1984, 1986, 1990b; MARSH; KÖLLER, 2003; MARSH; O’MARA, 2008; SOUZA; BRITO 2008).

\section{Atribuições de causalidade para o sucesso e para o fracasso}

As atribuições de causalidade envolvem as explicações ou causas que o aluno atribui ao seu desempenho (seja ele de sucesso ou fracasso), tais como capacidade, esforço e causas externas. As causas levantadas pelos alunos podem ser agrupadas a partir de três dimensões básicas: lócus, estabilidade e controlabilidade. A primeira dimensão refere-se à localização da causa como interna ou externa ao indivíduo. A estabilidade remete à imutabilidade da causa através do tempo. A controlabilidade diz respeito à extensão em que a causa está sujeita à interferência do indivíduo Dessa forma, pode-se considerar que a capacidade é uma atribuição interna, estável e incontrolável, que o esforço é uma atribuição interna, instável e controlável, e, por fim, que as causas externas são externas, instáveis e incontroláveis (PINTRICH; SCHUNK, 2002; WEINER, 1985).

Os estudos indicam que os alunos que atribuem o sucesso à capacidade e ao esforço têm maior probabilidade de obter um bom rendimento acadêmico do que os alunos que atribuem o sucesso a causas externas (GRAHAM; WEINER, 1996; MARSH, 1984, 1986; NÚÑEZ et al., 2005; WEINER, 1985).

\section{Metas de realização acadêmica}

As metas de realização referem-se aos propósitos que um indivíduo espera alcançar. Elas envolvem um conjunto de crenças, atribuições e afetos que influenciam a forma como o indivíduo se engaja nas atividades escolares (AMES, 1992; BZUNECK, 2009b).
A orientação de meta aprender refere-se ao propósito do indivíduo de desenvolvimento de competência (AMES, 1992). Os alunos orientados por essa meta focalizam-se na aprendizagem, no entendimento, no desenvolvimento de habilidades e no domínio de informações. Pode-se considerar que a meta aprender envolve um propósito de desenvolvimento e crescimento pessoal que norteia os comportamentos de realização envolvidos no engajamento em tarefas escolares (AMES, 1992; AMES; ARCHER, 1988; ELLIOT; DWECK, 1988; PINTRICH; SCHUNK, 2002).

Os estudos têm mostrado que os alunos que adotam a meta aprender relatam altos níveis de envolvimento na tarefa (HARACKIEWICZ et al., 2002), bem como uso de estratégias de aprendizagem que aumentam o entendimento conceitual e a lembrança das informações (AMES; ARCHER, 1988; ELLIOT; MCGREGOR, 2001; GREENE; MILLER, 1996; MEECE; BLUMENFELD; HOYLE, 1988; PINTRICH; SCHUNK, 2002; WOLTERS, 2004).

A orientação de meta performance-evitação refere-se ao propósito de demonstração de competência, evitando-se criar uma impressão de baixa capacidade (AMES, 1992; AMES; ARCHER, 1988; ELLIOT; DWECK, 1988; PINTRICH; SCHUNK, 2002). Esse tipo de meta está associado a resultados negativos, tais como baixa autoeficácia, ansiedade, esquiva de estratégias de busca de ajuda e baixas notas (ELLIOT, 1999; PINTRICK; SCHUNK, 2002).

Os alunos orientados pela meta performance-aproximação focalizam-se no manejo da impressão que os outros têm de sua capacidade, tentando criar uma impressão de alta capacidade. Para esses alunos, o sucesso é ter sua capacidade reconhecida publicamente e estar entre os melhores no meio acadêmico (PINTRICH, 2003; PINTRICH; SCHUNK, 2002).

Os resultados relativos aos efeitos da meta performance-aproximação sobre o rendimento acadêmico são inconclusivos. Estudos realizados em situações mais competitivas, como as experienciadas por alunos de ensino 
superior, indicam que os alunos que adotam tal meta podem apresentar resultados positivos tais como persistência, afeto positivo e notas mais elevadas (BARRON; HARACKIEWICZ; 2001; HARACKIEWICZ et al., 2002; LUO et al., 2011; PINTRICH, 2003).

Os efeitos da adoção desse tipo de meta por parte de alunos de ensino fundamental sobre o rendimento acadêmico ainda não são claros. Os estudos demonstram que essa orientação de metas, por relacionar-se a uma necessidade de demonstração da capacidade, também se associa a resultados negativos, tais como ansiedade, afeto negativo seguindo o fracasso, comportamento disruptivo e baixa retenção de conhecimentos (AMES; ARCHER, 1988; KAPLAN; MAEHR, 2007; GREENE; MILLER, 1996; PINTRICH; SCHUNK, 2002).

Uma das preocupações atuais manifestada por professores e autoridades da área educacional refere-se ao insatisfatório domínio das habilidades e dos conhecimentos previstos de serem adquiridos no ensino fundamental. A baixa qualidade da aprendizagem e o baixo rendimento escolar dos estudantes têm sido evidenciados pelos resultados obtidos em avaliações governamentais como o Sistema de Avaliação da Educação Básica, a Prova Brasil e o Índice de Desenvolvimento da Educação Básica.

A falta de motivação é comumente utilizada pelos professores como uma das explicações para os resultados negativos quanto à aprendizagem e ao aproveitamento acadêmico de parcela significativa dos alunos. Uma das limitações do uso de explicações sobre a presença ou ausência de motivação é o favorecimento de uma visão da motivação como um traço global e estável que o aluno tem ou não tem.

No âmbito das teorias cognitivo-sociais, enfatiza-se o exame da qualidade da motivação em termos de como se apresentam os aspectos cognitivos e os aspectos contextuais que influenciam o engajamento motivacional dos alunos, sendo levada em conta a interação do aluno com o ambiente de sala de aula e da escola para a promoção de um engajamento motivacional que favoreça a aprendizagem e o rendimento. Em consonância com essa perspectiva teórica, o presente estudo pretendeu caracterizar a motivação de um grupo de estudantes em termos de três dos principais aspectos cognitivos da motivação - autoconceito, atribuições de causalidade e metas de realização -, bem como identificar relações entre autoconceito, atribuições de causalidade e metas, e rendimento alto, médio e baixo na disciplina de português.

\section{Método}

\section{Participantes}

Participaram deste estudo 159 alunos (80 do sexo masculino e 79 do sexo feminino) cursando duas classes de $6^{\text {a }}$ série $(\mathrm{N}=41)$ e quatro classes de $7^{\text {a }}$ série $(\mathrm{N}=118)$ do ensino fundamental de uma escola pública de um município de porte médio do interior do Estado de São Paulo. A idade dos participantes variou de 12 a 15 anos, sendo a média de 13 anos.

Os alunos foram divididos em três grupos em razão do rendimento do aluno na disciplina de língua portuguesa, sendo tal rendimento estabelecido a partir dos conceitos obtidos na disciplina nos três primeiros bimestres do ano letivo em que ocorreu a coleta de dados. Os conceitos foram transformados em números: $\mathrm{A}=5, \mathrm{~B}=4, \mathrm{C}=3, \mathrm{D}=2$ e $\mathrm{E}=1$. Para calcular o valor do rendimento de cada aluno, foram somados os valores dos três primeiros bimestres.

A partir dos valores de todos os alunos, foram obtidos os quartis dos valores de rendimento por meio do programa estatístico SPSS 11.5, de modo que tais valores foram divididos em quatro intervalos: um intervalo com 25\% dos valores mais baixos, dois intervalos formados por $25 \%$ dos valores de rendimento intermediários e um intervalo com 25\% dos valores de desempenho mais altos.

Dessa forma, o critério para a classificação em um dos três grupos de rendimento foi o intervalo em que esse valor se encontrava. No primeiro quartil, correspondente ao valor de 
rendimento menor ou igual a 8, foram classificados os alunos com rendimento acadêmico baixo $(\mathrm{N}=42)$. No segundo e no terceiro quartis, correspondentes aos valores de rendimento entre 9 e 11, foram classificados os alunos com rendimento acadêmico médio $(\mathrm{N}=65)$, e no quarto quartil, correspondente ao valor de rendimento acima de 12, foram classificados os alunos com rendimento acadêmico alto $(N=52)$.

\section{Aspectos éticos}

A pesquisa foi autorizada pelo Comitê de Ética em Pesquisa em Seres Humanos da Universidade Federal de São Carlos (processo CEP-UFSCar $n^{\circ}$ 132/04). A formalização do consentimento se deu por meio de um termo de consentimento livre e esclarecido assinado pelos alunos e pelos pais ou responsáveis legais.

\section{Instrumentos}

Escala de Metas Pessoais dos Alunos: esta escala faz parte do instrumento PALS - Patterns of Adaptative Learning Scales (MIDGLEY et al., 2000) e vem sendo amplamente utilizada em pesquisas internacionais para avaliar as metas pessoais dos alunos de ensino fundamental e médio. 0 instrumento foi traduzido e adaptado para a população brasileira por Zambon (2006). Ele contém catorze afırmações referentes às percepções dos alunos quanto aos seus propósitos perante as aulas e atividades da disciplina de português. Cada um dos itens pode ser respondido de acordo com uma escala Likert de cinco pontos (definitivamente não é verdade; pouco verdade; é meio verdade; verdade; muito verdade).

$\mathrm{Na}$ escala da meta aprender (cinco itens), as afirmações referem-se a razões ou propósitos como: melhorar conhecimentos e habilidades; progredir; dominar sempre mais os conteúdos, com inovação e criatividade; aprender algumas novidades; entender sua tarefa; aprender o máximo possível. Na escala da meta performance-aproximação (cinco itens), as afırmações fazem referência a impressionar os outros e a agradar o professor. Já na escala da meta performance-evitação (quatro itens), as afırmações abordam aspectos como evitar o fracasso, evitar parecer incapaz ou evitar estar entre os mais fracos da sala. Foi realizado um teste de consistência interna para os dados coletados por ocasião deste estudo. Para a subescala que agrega os itens referentes à meta aprender, o coeficiente de confiabilidade foi de 0,80. Para a subescala que contém os itens relativos à meta performance-aproximação, o coeficiente de confiabilidade foi de 0,80 . Para a subescala referente à meta performance-evitação, o coeficiente de confiabilidade foi de 0,64. Todos os itens apresentaram a correlação item-total acima de 0,20.

Escala de Atribuição de Causalidade para Atividades de Leitura e Escrita de Textos (EACALE): o propósito deste instrumento é medir as percepções dos alunos em relação às causas de seu sucesso ou fracasso em atividades relacionadas à disciplina de português no segundo ciclo do ensino fundamental. 0 instrumento aplicado consiste em uma versão desenvolvida por Cabau e De Rose (2004) com base na Escala de Atribuição de Sidney (Sydney Attribution Scale - SAS), de Marsh (1984). 0 instrumento é composto por doze situações hipotéticas relativas à leitura/escrita características do segundo ciclo do ensino fundamental envolvendo combinações de dois resultados hipotéticos (situações de sucesso e fracasso) e três tipos de causas (capacidade, esforço e causas externas), totalizando 36 itens. Para cada situação, o aluno tem de avaliar as três possíveis causas (capacidade, esforço e causas externas) e responder a cada uma considerando cinco opções (falso; quase falso; às vezes falso; às vezes verdadeiro; quase verdadeiro; verdadeiro).

Foi realizado um teste de consistência interna para os dados coletados por ocasião deste estudo. Para a subescala referente a sucesso/ capacidade, o coeficiente de confiabilidade foi 
de 0,75 ; para a subescala referente a sucesso/ esforço, o coeficiente de confiabilidade foi de 0,76 ; para a subescala referente a sucesso/causas externas, o coeficiente de confiabilidade foi de 0,51 ; para a subescala referente a fracasso/capacidade, o coeficiente de confiabilidade foi de 0,63 ; para a subescala relativa a fracasso/esforço, o coeficiente de confiabilidade foi de 0,57; e para a subescala referente a fracasso/causas externas, o coeficiente de confiabilidade foi de 0,44.

SDQ II - Self-Description Questionnaire II (MARSH, 1990a): o questionário de autodescrição, traduzido para o português e adaptado para a população brasileira por Zambon e De Rose (2003), consiste em um inventário de 102 itens de autorrelato subdivididos em onze escalas, que acessam três áreas de autoconceito acadêmico (matemática, português e autoconceito escolar geral), seis áreas de autoconceito não acadêmico (habilidades físicas, aparência física, relações com o sexo oposto, relações com o mesmo sexo, relação com os pais, honestidade-lealdade, estabilidade emocional) e uma classe de autoconceito geral (que acessa o autovalor e a autossatisfação). Solicita-se que os adolescentes respondam a cada item utilizando uma escala Likert de seis pontos (falso; quase sempre falso; mais vezes falso do que verdade; mais vezes verdade do que falso; quase sempre verdade; verdade). Por ocasião deste estudo, foram utilizadas apenas as seguintes escalas acadêmicas do SDQ-II: escolar geral e português. Os itens que avaliam o autoconceito escolar geral (nove itens) referem-se à percepção de habilidades, capacidades e interesse nas matérias da escola em geral. Os itens que avaliam o autoconceito em português (dez itens) referem-se a percepções de capacidade e interesse em leitura, bem como a percepções das habilidades verbais e de capacidade e interesse em atividades verbais. Realizou-se um teste de consistência interna para os dados coletados por ocasião deste estudo e obtiveram-se os seguintes coeficientes de confiabilidade: 0,88 para a área escolar geral e 0,84 para português.

\section{Procedimento de coleta de dados}

A coleta foi realizada nas salas em que as seis turmas de alunos estavam tendo aula. A aplicação dos instrumentos ocorreu de forma coletiva e simultaneamente nas seis turmas 0s instrumentos foram aplicados por seis assistentes voluntários, devidamente instruídos quanto aos cuidados para a aplicação.

A coleta de dados ocorreu em dois dias subsequentes. No primeiro dia, foram coletados os dados referentes às metas de realização e às atribuições de causalidade; posteriormente, foram coletados os dados referentes ao autoconceito. A duração média de cada uma das aplicações foi de cinquenta minutos.

Inicialmente, os aplicadores leram em voz alta as instruções para o preenchimento de cada um dos instrumentos, informando aos alunos que poderiam esclarecer quaisquer dúvidas durante a aplicação.

\section{Tratamento e análise dos dados}

Os escores da Escala de Metas Pessoais dos Alunos foram obtidos a partir da soma dos escores de cada item para cada uma das três subscalas (meta aprender, meta performance-aproximação e meta performance-evitação). Os valores das respostas eram os seguintes: definitivamente não é verdade $=1$ ponto; pouco verdade $=2$ pontos; é meio verdade $=3$ pontos; verdade $=4$ pontos; muito verdade $=5$ pontos. Para as subescalas aprender e performance-aproximação, com cinco itens cada uma, a pontuação mínima é de 5 pontos e a máxima é de 25 pontos. Para a subescala performance-evitação, com quatro itens, a pontuação mínima é de 4 pontos e a máxima é de 20 pontos.

Os escores das atribuições obtidos na EACALE foram calculados pela soma das respostas para os seis itens que compunham cada subescala: sucesso/capacidade; sucesso/esforço; sucesso/causas externas; fracasso/habilidade; fracasso/esforço; fracasso/causas externas. Os valores das respostas eram: falso $=1$ ponto; 
maioria falso $=2$ pontos; às vezes falso, às vezes verdadeiro $=3$ pontos; maioria verdadeiro $=$ 4 pontos; verdadeiro $=5$ pontos. Dessa forma, a pontuação mínima de um aluno em cada subescala é 6 e a máxima 30.

As respostas dadas ao SDQ-II foram calculadas de acordo com a seguinte pontuação: falso = 1 ponto; quase sempre falso $=2$ pontos; mais vezes falso do que verdade $=3$ pontos; mais vezes verdade do que falso $=4$ pontos; quase sempre verdade $=5$ pontos; verdade $=6$ pontos. De acordo com o manual do instrumento original, a pontuação dos itens que expressavam um autoconceito negativo era invertida, subtraindo-se o valor do item de 7; por exemplo, se o aluno respondeu quase sempre falso (2 pontos) a um desses itens, sua pontuação final é de 5 pontos $(7-2=5)$. Para calcular o escore em cada uma das subescalas de autoconceito, foram somados os valores dos itens correspondentes. Para a subescala escolar geral, a pontuação poderia variar de 9 a 54 pontos. Para as subescalas referentes a língua portuguesa e matemática, a pontuação poderia variar de 10 a 60 pontos.

Depois de os valores das respostas de todos os participantes terem sido computados no
Programa SPSS 11.5, foi feita a análise dos dados. A primeira etapa envolveu uma análise descritiva dos escores das metas de orientação, atribuição de causalidade e autoconceito de cada grupo de desempenho acadêmico, resultando nos seguintes valores: médias e desvios padrões.

$\mathrm{Na}$ segunda etapa, as médias de cada uma das variáveis obtidas pelos diferentes grupos foram comparadas por meio da realização do Teste ANOVA, no qual se considerou um nível de significância estatística de $\mathrm{p}<0,05$.

\section{Resultados}

Na Tabela 1, são apresentados os resultados quanto aos escores médios e aos desvios padrões para as áreas de autoconceito avaliadas (escolar geral e português), as três possíveis atribuições de causalidade para o sucesso (capacidade, esforço e causas externas), as três possíveis atribuições de causalidade para o fracasso (capacidade, esforço e causas externas) e as três metas avaliadas (aprender, performance-aproximação e performance-evitação) obtidas pelo grupo de alunos com rendimento alto (GA), pelo grupo com rendimento médio (GM) e pelo grupo com rendimento baixo (GB).

Tabela 1 - Média e desvio padrão (dp) dos escores médios nos itens das áreas de autoconceito, atribuições de causalidade e metas de orientação pessoal apresentados pelos grupos com rendimento acadêmico baixo, médio e alto

\begin{tabular}{|c|c|c|c|c|c|c|c|}
\hline & & \multicolumn{2}{|c|}{ GA } & \multicolumn{2}{|c|}{ GM } & \multicolumn{2}{|c|}{ GB } \\
\hline & & média & $\mathrm{dp}$ & média & $\mathrm{dp}$ & média & $\mathrm{dp}$ \\
\hline \multirow{2}{*}{$\begin{array}{c}\text { Autoconceito } \\
(\text { mín. }=1 ; \text { máx. }=6)\end{array}$} & Escolar geral & 5,20 & 0,91 & 4,33 & 1,05 & 3,37 & 1,19 \\
\hline & Português & 5,02 & 0,74 & 4,26 & 1,03 & 3,56 & 1,23 \\
\hline \multirow{6}{*}{$\begin{array}{l}\text { Atribuições causais } \\
(\text { mín.=1; máx. }=5)\end{array}$} & Sucesso/capacidade & 3,82 & 0,61 & 3,50 & 0,89 & 3,18 & 0,97 \\
\hline & Sucesso/esforço & 4,12 & 0,76 & 3,91 & 0,79 & 3,68 & 1,00 \\
\hline & Sucesso/causas externas & 2,23 & 0,70 & 2,51 & 0,79 & 2,67 & 0,71 \\
\hline & Fracasso/capacidade & 1,71 & 0,62 & 2,10 & 0,83 & 2,24 & 0,82 \\
\hline & Fracasso/esforço & 2,77 & 0,78 & 2,96 & 0,88 & 2,94 & 0,83 \\
\hline & Fracasso/causas externas & 2,42 & 0,94 & 2,38 & 0,90 & 2,58 & 1,08 \\
\hline \multirow{3}{*}{$\begin{array}{c}\text { Metas } \\
\text { de realização } \\
(\text { mín. }=1 ; \text { máx. }=5)\end{array}$} & Aprender & 4,51 & 0,43 & 4,19 & 0,56 & 3,82 & 0,81 \\
\hline & Performance-aproximação & 2,59 & 1,02 & 2,67 & 0,84 & 2,46 & 0,79 \\
\hline & Performance-evitação & 2,72 & 1,10 & 2,88 & 0,80 & 2,71 & 0,80 \\
\hline
\end{tabular}

Fonte: Dados da pesquisa. 
Com o objetivo de identificar possíveis diferenças entre os grupos com baixo, médio e alto rendimento acadêmico quanto às médias dos itens relativos a autoconceito, atribuições de causalidade e metas de realização, a Tabela 2 mostra o nível de significância estatística para os escores médios nos itens relativos a cada uma das variáveis investigadas nas comparações entre os grupos com rendimento baixo, médio e alto.

Tabela 2 - Nível de significância estatística da diferença dos escores médios nos itens das áreas de autoconceito, atribuições de causalidade e metas de realização apresentados na comparação dos diferentes grupos de rendimento

\begin{tabular}{|c|c|c|c|c|}
\hline \multirow{2}{*}{\multicolumn{2}{|c|}{ Variáveis }} & \multicolumn{3}{|c|}{ Grupos de rendimento } \\
\hline & & $\mathrm{GB} \times \mathrm{GM}$ & $\mathrm{GB} \times \mathrm{GA}$ & $\mathrm{GM} \times \mathrm{GA}$ \\
\hline \multirow{2}{*}{ Autoconceito } & Escolar geral & $0,00^{*}$ & $0,00^{*}$ & $0,00^{*}$ \\
\hline & Português & $0,00^{*}$ & $0,00^{*}$ & $0,00^{*}$ \\
\hline \multirow{6}{*}{ Atribuições causais } & Sucesso/capacidade & 0,14 & $0,00^{\star \star}$ & 0,12 \\
\hline & Sucesso/esforço & 0,39 & $0,04^{*}$ & 0,42 \\
\hline & Sucesso/causas externas & 0,58 & $0,02^{*}$ & 0,12 \\
\hline & Fracasso/capacidade & 0,68 & $0,00^{* *}$ & $0,02^{*}$ \\
\hline & Fracasso/esforço & 0,99 & 0,65 & 0,50 \\
\hline & Fracasso/causas externas & 0,57 & 0,73 & 0,96 \\
\hline \multirow{3}{*}{ Metas de realização } & Aprender & $0,00^{* *}$ & $0,00^{\star *}$ & 0,77 \\
\hline & Performance-aproximação & 0,47 & 0,77 & 0,87 \\
\hline & Performance-evitação & 0,64 & 0,99 & 0,65 \\
\hline
\end{tabular}

Post Hoc de Scheffè

Fonte: Dados da pesquisa.

${ }^{*} p<0,05 \quad{ }^{* *} p<0,01$

A comparação dos grupos com diferentes níveis de rendimento quanto ao autoconceito destaca diferenças significativas. 0 grupo com rendimento acima da média em português apresenta autoconceito mais positivo do que os demais, e o grupo com rendimento na média mostra autoconceito mais elevado do que o grupo com desempenho médio em leitura.

Foram verificadas diferenças significativas quanto aos valores dos escores médios das atribuições de capacidade e esforço para o sucesso entre o grupo com alto rendimento e o com rendimento médio, sendo os valores do GA superiores aos do GM. Também se observaram diferenças signifıcativas entre os valores médios da atribuição a causas externas para o sucesso entre o grupo com alto e o grupo com baixo rendimento, sendo os valores do GB superiores aos do GA.

Constataram-se diferenças significativas quanto aos valores dos escores médios da atribuição de capacidade para o fracasso entre o grupo com alto rendimento e os grupos com rendimento médio e baixo, sendo os valores obtidos pelo GM e pelo GB superiores aos obtidos pelo GA.

Com relação à meta aprender, o GA apresentou média significativamente mais elevada do que o GM e o GB. 0 GM apresentou média significativamente maior do que o GB. 
Não foram observadas diferenças estatisticamente significativas entre os três grupos em relação às metas performance-aproximação e performance-evitação.

\section{Discussão}

0 presente estudo pretendeu caracterizar a motivação de um grupo de estudantes em termos de três dos principais aspectos cognitivos - autoconceito, atribuições de causalidade e metas de realização -, bem como identificar relações entre autoconceito, atribuições de causalidade e metas, e rendimento alto, médio e baixo na disciplina de português.

Os resultados das comparações do autoconceito acadêmico, das atribuições de causalidade para o sucesso e o fracasso e as metas de realização dos alunos indicaram diferenças significativas entre alunos com alto rendimento e alunos com rendimentos médio e baixo. Os escores médios dos alunos com notas baixas e médias relativos aos aspectos motivacionais foram menos positivos. Eles apresentaram um autoconceito mais baixo, tenderam a atribuir o sucesso mais a causas externas e o fracasso mais à capacidade; além disso, adotaram menos a meta aprender do que o grupo com rendimento alto. Em contrapartida, os alunos com notas altas apresentaram autoconceitos em leitura e escrita mais elevados, atribuíram mais o sucesso à capacidade e ao esforço e demonstraram maior tendência à adoção da meta aprender do que os alunos com notas médias e baixas.

$\mathrm{Na}$ medida em que os resultados relativos a esse grupo de alunos do segundo ciclo do ensino fundamental de uma escola pública brasileira são consonantes com os resultados encontrados em pesquisas internacionais prévias, pode-se considerar que este estudo contribui para ampliar a generalidade dos achados que mostram a importância da motivação para o aproveitamento acadêmico dos alunos. Os resultados são comparáveis com os estudos que buscaram verificar as associações entre rendimento acadêmico e autoconceito (CARNEIRO;
MARTINELLI; SISTO, 2003; GREENE; MILLER, 1996; NÚÑ̃Z et al., 2005), a atribuição de sucesso ao esforço e à capacidade (ECCLES; WIGFIELD, 2002; GRAHAM; WEINER, 1996; MARSH, 1986, 1984; PAIVA; BORUCHOVITCH, 2010; WEINER, 1985) e a meta aprender (AMES; ARCHER, 1988; ELLIOT; DWECK, 1988; GREENE; MILLER, 1996; HARACKIEWICZ et al., 2002; WOLTERS, 2004; ZENORINI; SANTOS; MONTEIRO, 2011).

A proposta do estudo de caracterizar a motivação no contexto escolar à luz das contribuições teóricas e das evidências empíricas derivadas das atuais teorias motivacionais possibilitou levar em conta os múltiplos aspectos cognitivos que a influenciam, obtendo-se uma descrição abrangente e diferenciada de como tais dimensões apresentam-se para os alunos com baixo, médio e alto rendimento em português.

Em relação à qualidade da motivação, os resultados podem ser discutidos em termos do que eles apontam quanto à qualidade do engajamento motivacional dos alunos. Em geral, a análise do engajamento motivacional abrange os seguintes componentes: a escolha pelo envolvimento em determinada tarefa acadêmica ou não, o engajamento comportamental e a persistência nas realizações acadêmicas. 0 engajamento comportamental pode variar quanto à quantidade de esforço empregado nas tarefas em aula, por meio de apresentação de comportamentos observáveis, tais como fazer anotações durante as aulas, fazer questões durante e após as aulas, discutir as ideias abordadas nas aulas com os colegas, dedicar uma razoável quantidade de tempo ao estudo e à preparação para as aulas e provas, e buscar informações complementares sobre o que está sendo estudado. A persistência nas tarefas é inferida quando o aluno continua tentando realizá-la mesmo diante de dificuldades, cansaço ou tédio (BZUNECK, 2009a; PINTRICH; SCHUNK, 2002).

Os estudos mostram que o autoconceito influencia o aluno a engajar-se em tarefas novas e desafiadoras e a persistir nas atividades, uma vez que o indivíduo tende a escolher 
sistematicamente experiências e ambientes que sejam consistentes com suas crenças sobre si mesmo, o que, por sua vez, tem efeitos sobre como as tarefas acadêmicas são executadas e sobre o desempenho acadêmico (CARNEIRO; MARTINELLI; SISTO, 2003; CIA; BARHAM, 2008; MARSH, 1984, 1986, 1990b; MARSH; KÖLLER, 2003; MARSH; O'MARA, 2008; SOUZA; BRITO 2008).

Os estudos sobre a atribuição de causalidade têm mostrado que as causas mais frequentemente utilizadas pelos alunos para explicarem o sucesso e o fracasso em tarefas acadêmicas estão associadas a diferentes resultados psicológicos e motivacionais, tais como expectativas de sucesso futuro, autoeficácia, sentimentos positivos e negativos quanto a si e quanto às tarefas relacionadas à aprendizagem. Esses resultados, por sua vez, mostram-se associados à escolha das atividades, à persistência, ao esforço dispendido nas tarefas e aos processos relevantes para motivação e aprendizagem. Dessa forma, considera-se que, indiretamente, as atribuições afetam o engajamento cognitivo e o aproveitamento acadêmico dos alunos (ECCLES; WIGFIELD, 2002; GRAHAM; WEINER, 1996; MARSH, 1986, 1984 PAIVA; BORUCHOVITCH, 2010; WEINER, 1985).

Os estudos têm mostrado que os alunos que adotam a meta aprender persistem mais em tarefas difíceis (ELLIOT; DWECK, 1988), relatam altos níveis de esforço (AMES; ARCHER, 1988; ELLIOT; DWECK, 1988; GREENE; MILLER, 1996; PINTRICH; SCHUNK, 2002), sentem orgulho e satisfação quanto ao sucesso decorrente de esforço, sentem-se culpados quanto à falta de esforço, bem como apresentam atitudes positivas quanto à aprendizagem e um interesse intrínseco em aprender (PINTRICH; SCHUNK, 2002).

Em suma, a pesquisa sobre o engajamento motivacional tem indicado que os alunos que apresentam autoconceito positivo, que adotam a meta aprender e que atribuem o sucesso ou o fracasso a causas internas, instáveis e controláveis (esforço) estão mais propensos a exibirem formas de engajamento motivacional positivo a curto prazo (engajam-se mais em tarefas de aprendizagem, empregam mais esforço, persistem mais e procrastinam menos) e engajamento a longo prazo (escolhem participar de aulas adicionais da matéria) (GREENE; MILLER, 1996; KAPLAN; MAEHR, 2007; NUÑES et al., 2005; PAJARES; BRITNER; VALIANTE, 2000).

Também se tem demonstrado que os alunos que apresentam autoconceito negativo adotam as metas performance-aproximação e performance-evitação, atribuem o sucesso ou o fracasso a causas internas, estáveis e incontroláveis (capacidade) e apresentam tendência a fazerem menos escolhas de envolvimento em tarefas de aprendizagem, a empregarem menos esforço, a serem menos persistentes, a procrastinarem mais, a desistirem quando a tarefa mostra-se mais difícil e a apresentarem menor interesse em atividades extras (GREENE; MILLER, 1996; KAPLAN; MAEHR, 2007; NUÑES et al., 2005; PAJARES; BRITNER; VALIANTE, 2000).

A partir dos resultados da presente investigação, serão feitas considerações quanto a diferenças na qualidade da motivação dos alunos com baixo, médio e alto rendimento na disciplina de português, na medida em que eles exibiram diferentes padrões motivacionais (variações no autoconceito, nas atribuições de causalidade e nas metas de realização). Os padrões motivacionais podem ser considerados como adaptativos ou não adaptativos em função do quanto favorecem ou comprometem o engajamento motivacional, a aprendizagem e o rendimento. Pode-se inferir que os alunos deste estudo que alcançaram um nível de rendimento acadêmico acima da média na disciplina de português apresentaram padrões motivacionais adaptativos. Os alunos que alcançaram níveis médios e baixos de rendimento apresentaram padrões motivacionais não adaptativos em termos de favorecimento do engajamento motivacional, da aprendizagem e do rendimento.

Várias implicações podem ser apontadas a partir do exame dos padrões motivacionais observados entre os alunos à luz do entendimento de que eles têm um caráter mutável e são 
sensíveis à influência do ambiente da sala de aula e das práticas dos professores. A pesquisa sobre motivação no contexto escolar apresenta subsídios consistentes sobre como o professor pode influenciar a motivação do aluno, seja de forma positiva, seja de forma negativa (AMES, 1992; PINTRICH; SCHUNK, 2002; PINTRICH, 2003; STIPEK, 1996). Para os alunos que apresentaram padrões motivacionais não favorecedores de motivação e rendimento, um investimento poderia ser feito pelos professores no sentido de um aprimoramento de seus padrões motivacionais. Para os alunos que apresentam padrões motivacionais adaptativos, o investimento poderia ser dirigido para a manutenção dos resultados.

Considerações sobre as possibilidades de promoção e melhoria na qualidade da motivação dos alunos podem ser feitas à luz do conhecimento produzido sobre como os professores e as práticas de ensino influenciam a motivação dos alunos tanto de forma positiva como negativa. Dentre tais considerações, estão: favorecer o autoconceito; estimular os alunos a utilizarem o esforço como atribuição para explicar o sucesso e o fracasso acadêmico; e favorecer que os alunos adotem a meta aprender. Essa possibilidade será ilustrada a partir das contribuições advindas da pesquisa sobre metas de realização.

Os estudiosos das metas de realização recomendam que, no contexto escolar, a adoção da meta aprender pelos alunos deveria ser fortalecida por ser considerada mais adaptativa e favorecedora da aprendizagem; além disso, eles oferecem um referencial para demonstrar como os professores afetam as metas que os alunos adotam na sala de aula e apontar práticas que favoreçam que os alunos adotem a meta aprender (AMES, 1992; MIGDLEY et al., 1998; MIDGLEY; KAPLAN; MIDDLETON, 2001; PINTRICK; SCHUNK, 2002; ZENORINI; SANTOS; MONTEIRO, 2011).

Eles sugerem que os comportamentos e o discurso dos professores, em geral, comunicam aos alunos suas crenças sobre os propósitos de realização, ou seja, que a estrutura de meta da sala de aula pode influenciar metas pessoais, comportamentos relacionados à realização, cognição e sentimentos dos alunos quanto à aprendizagem (AMES, 1992; MIDGLEY; KAPLAN; MIDDLETON, 2001; TURNER et al., 2003).

Os estudos relativos às metas de realização têm abordado um esquema de seis dimensões da estrutura da sala de aula que favorecem a meta aprender: o planejamento das tarefas da sala de aula ou das atividades; a distribuição de autoridade entre professor e alunos; o reconhecimento do esforço; as oportunidades para os alunos trabalharem com os outros em grupos; as formas de avaliação da aprendizagem; e o planejamento do tempo (AMES, 1992; PINTRICH; SCHUNK, 2002; TURNER et al., 2003).

A pesquisa sugere que, dependendo das práticas de ensino dos professores relacionadas a essas seis dimensões na sala de aula, os estudantes podem perceber a estrutura de metas como sendo mais ou menos orientada para aprender ou para a performance. Os professores, por exemplo, podem enfatizar o entendimento e o progresso pessoal (favorecendo um ambiente de sala de aula orientado para o aprender) ao fornecerem reconhecimento aos alunos pelo engajamento em tarefas acadêmicas desafiadoras e valorizarem o progresso obtido, considerando-o na avaliação. Eles podem enfatizar a competição (favorecendo um ambiente de sala de aula orientado para a performance) ao priorizarem o reconhecimento somente para aqueles com alto rendimento, por meio do encorajamento dos alunos a se sobressaírem em relação aos colegas, e ao utilizarem padrões normativos de avaliação (AMES, 1992; PINTRICK; SCHUNK, 2002; TURNER et al., 2003).

As descobertas empíricas dos estudos que examinam a influência das percepções dos alunos acerca da estrutura de meta da sala de aula têm indicado que a estrutura de meta aprender mostra-se mais facilitadora de resultados positivos de aprendizagem. Ames e Archer (1988) demonstraram que quando os alunos percebiam a estrutura de meta de suas salas de aula, havia maior probabilidade de relatarem o uso de estratégias de aprendizagem adaptativas, 
arriscarem a envolver-se em tarefas desafiadoras, relatarem maior satisfação com a aula e acreditarem que o sucesso é devido ao esforço.

Concluindo, é importante salientar a relevância de se conhecer e descrever de forma precisa os aspectos cognitivos da motivação dos alunos, uma vez que eles fornecem indicadores da qualidade da motivação, bem como a identificação de padrões motivacionais adaptativos e não adaptativos. Os achados do presente estudo contribuem para destacar a importância do investimento na promoção da motivação dos alunos como uma condição fundamental para a melhoria do rendimento acadêmico. Por outro lado, considera-se uma limitação do estudo o fato de sua ênfase ter recaído somente na relação entre os aspectos cognitivos e o rendimento acadêmico. Constata-se ser necessário que o delineamento de pesquisas futuras avance envolvendo o exame conjunto tanto dos aspectos cognitivos, quanto dos aspectos contextuais da motivação no ambiente escolar. 


\section{Referências}

AMES, Carole. Classrooms: goals, structures, and student motivation. Journal of Educational Psychology, v. 84, n. 3, p. 261-271, 1992.

AMES, Carole; ARCHER, Jennifer. Achievement goals in the classroom: students' learning strategies and motivation process. Journal of Educational Psychology, v. 80, n. 3, p. 260-267, 1988.

BARRON, Kenneth E.; HARACKIEWICZ, Judith M. Achievement goals and optimal motivation: testing multiple goal models. Journal of Educational Psychology, v. 80, n. 5, p. 706-722, 2001.

BZUNECK, José Aloyseo. A motivação do aluno: aspectos introdutórios. In: BORUCHOVITCH, Evely; BZUNECK, José Aloyseo (Orgs.). A motivação do aluno: contribuições da psicologia contemporânea. 4. ed. Petrópolis: Vozes, 2009a. p. 9-36.

A motivação do aluno orientado a metas de realização. In: BORUCHOVITCH, Evely; BZUNECK, José Aloyseo (Orgs.). A motivação do aluno: contribuições da psicologia contemporânea. 4. ed. Petrópolis: Vozes, 2009b. p. 58-77.

CABAU, Priscila M. P. Ferraz; DE ROSE, Tânia M. Santana. A motivação acadêmica de adolescentes: um estudo das metas de realização e atribuições de causalidade. Monografia (Graduação em Psicologia) - Universidade Federal de São Carlos, São Carlos, 2004.

CARNEIRO, Gabriela R. da Silva; MARTINELLI, Selma de Cássia; SISTO, Fermino Fernandes. Autoconceito e dificuldades de aprendizagem. Psicologia: Reflexão e Crítica, Porto Alegre v. 16, n. 3, p. 427-434, 2003.

CIA, Fabiana; BARHAM, Elizabeth Joan. Estabelecendo relação entre autoconceito e desempenho acadêmico de crianças escolares. PSICO, Porto Alegre, v. 39, n. 1, p. 21-27, 2008.

ECCLES, Jacquelynne S.; WIGFIELD, Allan. Motivational beliefs, values and goals. Annual Review of Psychology, v. 53, p. 109-132, 2002.

ELLIOT, Andrew J. Approach and avoidance motivation and achievement goals. Educational Psychologist, v. 34, p. 169-189, 1999.

ELLIOTT, Elaine S.; DWEECK, Carol S. Goals: An approach to motivation and achievement. Journal of Personality and Social Psychology, v. 54, p. 5-12. 1988.

ELLIOT, A.; MCGREGOR, Holly A. A 2 × 2 achievement goals framework. Journal of Personality and Social Psychology, v. 80, p. 501-519, 2001.

GRAHAM, Sandra; WEINER, Bernard. Theories and principles of motivation. In: BERLINER, David C.; CALFEE, Robert (Eds.), Handbook of educational psychology. New York: Simon \& Schuster Macmillan. 1996. p. 64-84.

GREENE, Barbara A.; MILLER, Raymond B. Influences on achievement: goals, perceived ability and cognitive engagement. Contemporary Educational Psychology, v. 21, n. 2, p. 181-192, 1996.

HARACKIEWICZ, Judith M. et al. Revision of achievement goal theory: necessary and illuminating. Journal of Educational Psychology, v. 94, n. 3, p. 638-645, 2002.

KAPLAN, Avi; MAEHR, Martin L. The contribution and prospects of goal orientation theory. Educational Psychology Review, v. 19, p. 141-187, 2007.

LUO, Wenshu et al. Do performance goals promote learning? A pattern analysis of Singapore students' achievement goals. Contemporary Educational Psychology, v. 36, p. 165-176, 2011.

MARSH, Herbert W. Relations among dimensions of self-attribution, dimensions of self-concept and academic achievements. Journal of Educational Psychology, v. 76, n. 6, p. 1291-1308, 1984.

Self-serving effect (bias?) in academic attributions: Its relation to academic achievement and self-concept. Journal of Educational Psychology, v. 78 n. 3, p. 190-200, 1986.

Self-description questionnaire - II. San Antonio, TX: The Psychological Corporation, 1990a. 
The structure of academic self-concept: the Marsh/Shavelson model. Journal of Educational Psychology, v. 76, p. 3-32, 1990 b.

MARSH, Herbert W.; KÖLLER, Olaf. Bringing together two theoretical models of relations between academic self-concept and achievement. In: MARSH, Herbert W; CRAVEN, Ronda G. (Eds.). International advances in self research. Connecticut: D.M. Information Age Publishing, 2003.

MARSH, Herbert W.; O'MARA, Alison. Reciprocal effects between academic self-concept, self-esteem, achievement, and attainment over seven adolescent years: unidimensional and multidimensional perspectives of self-concept. Personality and Social Psychology Bulletin, v. 34, p. 542- 552, 2008.

MEECE, Judith; BLUMENFELD, Phyllis C.; HOYLE, Rick H. Students' goal orientation and cognitive engagement in classroom activities. Journal of Educational Psychology, v. 80, n. 4, p. 514-523, 1988.

MIDGLEY, Carol; KAPLAN, Avi; MIDDLETON, Michael J. Performance-approach goals: Good for what, for whom, under what circumstances, and at what cost? Journal of Educational Psychology, v. 93, p. 77-86, 2001.

MIDGLEY, Carol et al. The development and validation of scales assessing student's achievements goal orientations. Contemporary Educational Psychology, v. 23, p. 113-131, 1998.

MIDGLEY, Carol et al. Manual for the patterns of adaptative learning scales. Ann Harbor: The University of Michigan, 2000.

NúÑEZ, José Carlos et al. Subgroups of attributional profiles in students with learning dificulties and their relation to self-concept and acadmic goals. Learning Disabilities Research \& Practice, v. 20, n. 2, p. 86-97, 2005.

PAIVA, Mirella L. M. Fernandes; BOUCHOVITCH, Evely. Orientações motivacionais, crenças educacionais e desempenho escolar de estudantes do ensino fundamental. Psicologia em Estudo, Maringá, v. 15, n. 2, p. 381-389, 2010.

PAJARES, Frank; BRITNER, Shari L.; VALIANTE, Giovanni. Relation between achievement goals and self-beliefs of middle school students in writing and science. Contemporary Educational Psychology, v. 25, p. 406-422, 2000.

PEIXOTO, Francisco; ALMEIDA, Leandro S. A organização do autoconceito: análise da estrutura hierárquica em adolescentes. Psicologia: Reflexão e Crítica, Porto Alegre, v. 24, n. 3, p. 533-541, 2011.

PINTRICH, Paul R. A motivational science perspective on the role of student motivation in learning and teaching contexts. Journal of Educational Psychology, v. 9, n. 4, p. 667-686, 2003.

PINTRICH, Paul R.; SCHUNK, Dale H. Motivation in education: theory, research and aplications. 2. ed. Upper Saddle River: Prentice Hall, 2002.

SHAVELSON, Richard J.; BOLUS, Roger. Self-concept: the interplay of theory and methods. Journal of Educational Psychology, v. 74, n. 1, p. 3-17, 1982

SOUZA, Liliane F.N. Inglez de; BRITO, Márcia R. Ferreira de. Crenças de auto-eficácia, autoconceito e desempenho em matemática. Estudos de Psicologia, Campinas, v. 25, n. 2, p. 193-201, 2008.

STIPEK, Deborah. Motivation and instruction. In: BERLINER, David C; CALFEE, Robert (Eds.). Handbook of educational psychology. New York: Simon \& Schuster Macmillan, 1996. p. 85-113.

TURNER, Julianne C. et al. Teacher discourse and sixth graders' reported affect and achievement behavior in two high-mastery/ highperformance mathematics classrooms. Elementary School Journal, v. 103, p. 357-382, 2003.

WEINER, Bernard. An attributional theory of achievement motivation and emotion. Psychological Review, v. 92, n. 4, p. 548-573, 1985.

WIGFIELD, Allan et al. Development of achievement motivation. In: DAMON, William; LERNER, Richard M. (Eds.). Handbook of child psychology. 6. ed. New York: Wiley, 2006. v. 3. 
WOLTERS, Christopher A. Advancing achievement goal theory: using goal structures and goal orientations to predict students' motivation, cognition, and achievement. Journal of Educational Psychology, v. 96, n. 2, p. 236-250, 2004.

ZAMBON, Melissa Picchi; DE ROSE, Tânia M. Santana de. Uma comparação racial da auto-estima e do autoconceito de adolescentes negros e brancos. Monografia (Graduação em Psicologia) - Universidade Federal de São Carlos, São Carlos, 2003.

ZAMBON, Melissa Picchi. Motivação de alunos: relações entre desempenho acadêmico, metas de realização, atribuições de causalidade e autoconceito acadêmico. Dissertação (Mestrado em Educação Especial) - Universidade Federal de São Carlos, São Carlos, 2006.

ZENORINI, Rita P. Campos; SANTOS, Acácia A. Angeli dos; MONTEIRO, Rebecca de Magalhães. Motivação para aprender: relação com o desempenho de estudantes. Paidéia, Ribeirão Preto, v. 21, n. 49, p. 157-164, 2011.

Recebido em: 31.01 .2012

Aprovado em: 06.08.2012

Melissa Picchi Zambon é psicóloga, mestre e doutoranda em Educação Especial pelo Programa de Pós-Graduação em Educação Especial da Universidade Federal de São Carlos (UFSCar). E-mail: melissapzambon@yahoo.com.br.

Tânia Maria Santana de Rose é psicóloga e professora do Departamento de Psicologia e do Programa de Pós-Graduação em Educação Especial da Universidade Federal de São Carlos (UFSCar). Coordena o Núcleo de Estudos de Motivação Acadêmica. 